\title{
October 2013 Critical Care Case of the Month: Slow to Respond
}

\author{
Michael P. Mohning, MD \\ Pulmonary Sciences and Critical Care Medicine \\ University of Colorado Hospital \\ Denver, CO
}

\section{History of Present IIIness}

A 66-year-old woman presents with confusion and lower extremity edema. She was brought to the emergency department by her family after 2-3 days of increasing confusion. She has fatigue and a dry non-productive cough but denies shortness of breath, chest pain, fevers or chills. She had a decrease in oral intake and constipation for several days.

\section{$P M H, S H, F H$}

Five months ago, she was admitted to a hospital for community acquired pneumonia and hyponatremia.

She is a never smoker, and doesn't use alcohol.

There is no significant family history.

\section{Medications}

- Omega 3 fatty acids

- Multivitamins

\section{Physical Examination}

Temperature $36.1^{\circ} \mathrm{C}$, blood pressure $106 / 61 \mathrm{~mm} \mathrm{Hg}$, heart rate 72 beats/min, respiratory rate 15 breaths/min, oxygen saturation $90 \%$ on room air.

She was confused, and oriented to self only. She had facial edema. Cardiac exam was normal. Pulmonary findings include rales at the lung bases. Her abdomen was non-tender, with active bowel sounds. She had 1+ lower extremity edema, no rashes, and delayed relaxation of reflexes.

\section{Laboratory}

She was anemic with hematocrit of $32 \%$, hemoglobin $11 \mathrm{~g} / \mathrm{dL}$ and WBC 5,000. Serum sodium is low at $118 \mathrm{meq} / \mathrm{L}$, anion gap was normal at 9 and potassium and calcium levels were normal. Albumin is low at $3.2 \mathrm{~g} / \mathrm{dL}$. Remaining liver function, blood glucose and creatinine are normal. EKG shows no T wave inversions or ST segment elevation. 


\section{Radiography}

Chest x-ray is shown in figure 1.

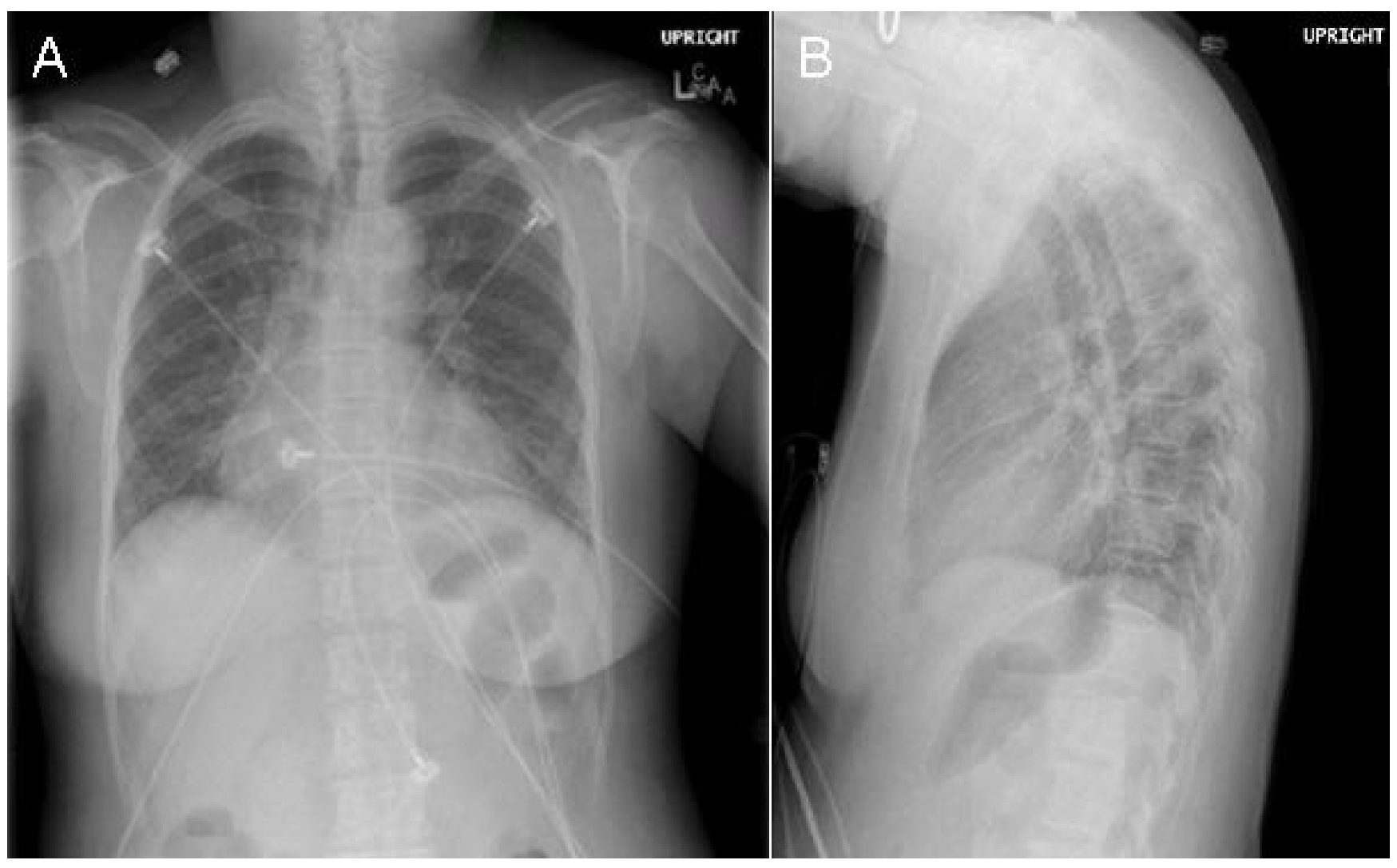

Figure 1. Admission PA (Panel A) and lateral (Panel B) chest X-ray.

Which best describes the chest-x-ray?

1. Bilateral interstitial infiltrates

2. Enlarged cardiac silhouette

3. Hyperexpanded lungs

4. Poor inspiratory effort

5. Pulmonary edema 


\section{Correct! \\ 4. Poor inspiratory effort}

Poor inspiratory effort often makes the lung bases appear to have an infiltrate. Although her heart silhouette approaches $50 \%$ of the chest diameter on PA film, this is hard to interpret for a less than full breath. With a full expansion, the silhouette would be less than $50 \%$ of the chest diameter. Her lungs are clearly not hyperexpanded.

A couple hours later, she becomes increasingly obtunded and is hypotensive.

She is transferred to the MICU and emergently intubated. Her pre-intubation ABG showed hypoventilation with pH 7.19, pCO2 59 mm Hg, pO2 210 mm Hg on 4 Lpm oxygen.

What further testing is indicated?

1. Blood cultures

2. Brain naturetic peptide (BNP)

3. Echocardiogram

4. Thyroid function testing

5. All of the above 


\section{Correct! \\ 5. All of the above}

For unexplained hypotension and mental status changes, looking for sepsis, infection, and myocardial ischemia or cardiomyopathy is appropriate. Because hypothyroidism can also cause obtundation and many of her presenting symptoms, this should be pursued. Blood cultures were sent, BNP level returned at 306 (normal range). An echocardiogram demonstrated normal LV ejection fraction, diastolic dysfunction, and the absence of valvular disease or an effusion. Her TSH level returned at $0.62 \mu \mathrm{IU} / \mathrm{mL}$, within the normal laboratory range. A random cortisol level returned at $<1.0 \mu \mathrm{g} / \mathrm{dL}$.

What would you do next?

1. ACTH stimulation test

2. Check thyroxine (T4) level

3. Treat with IV normal saline

4. 1 and 3

5. All of the above 


\section{Correct! \\ 5. All of the above}

Despite a normal TSH level, her free T4 level was low at $0.19 \mathrm{ng} / \mathrm{dL}$ (normal 0.5-1.9) and her total T3 was undetectable. An ACTH stimulation test showed lack of an appropriate increase to stimulation with cortisol values 30 min post stimulation: $4.1 \mu \mathrm{g} / \mathrm{dL}$ and 60 minutes post stimulation: $6.4 \mu \mathrm{g} / \mathrm{dL}$.

She was diagnosed with myxedema coma.

Myxedema coma is a life-threatening form of hypothyroidism with mortality approaching 40 to $50 \%(1,2)$. It is increasingly rare given the widespread screening for thyroid disease. It is most common in elderly women. Myxedema coma can be precipitated by infection, trauma, severe cold exposure and medications. Symptoms are nonspecific. Pulmonary and cardiac findings can include hypercapnia, hypoxia, laryngeal edema, bradycardia, pleural and pericardial effusions, and low cardiac output including shock. Metabolic manifestations include edema/anasarca, hyponatremia (up to $50 \%$ of cases), hypoglycemia, and hypothermia. Neurologic presentations can be those of lethargy, confusion, coma as seen here and even seizures. Constipation, abdominal pain, anorexia and nausea are common but nonspecific findings $(3,4)$.

Treatment of myxedema coma should include the following:

1. Stress doses of glucocorticoids

2. Supportive care

3. T4 by IV route

4. Triiodothyronine T3

5. All of the above 


\section{Correct! \\ 5. All of the above}

Experts debate whether both T3 and T4 are needed to acutely treat myxedema coma. The onset of action of T3 is quicker, bioavailability higher and most recommend use of both. Because of the rare situations where hypothyroidism is accompanied by adrenal insufficiency (as seen in this patient), glucocorticoids are recommended while awaiting lab testing $(3,4)$.

She was diagnosed with panhypopituitarism, myxedema coma and adrenal insufficiency. A magnetic resonance imaging MRI of the brain showed an atrophic appearance of the pituitary gland, thought due to prior ischemia or hemorrhage.

For this patient, myxedema coma with a normal TSH, low thyroxine, and associated panhypopituitarism with adrenal insufficiency was diagnosed as being from a hypothalamic or pituitary cause of the hypothyroid state, or central hypothyroidism. This is a rare etiology of hypothyroidism, seen in one of 1,000 hypothyroid patients (5). Causes include Infiltrating or compressive tumor such as a pituitary adenoma, or prior ischemia/infarct. The latter can be from pituitary apoplexy or post-partum pituitary necrosis, Sheehan syndrome.

What are the mechanisms of respiratory failure in myxedema coma?

1. Pulmonary embolism

2. Respiratory muscle weakness

3. Pulmonary edema

4. Blunted ventilatory drive

5. 1 and 3

6. 2 and 4 


\section{Correct!}

\section{2 and 4}

Ventilatory drives, both hypoxic and hypercapnic, are significantly blunted in the hypothyroid state and further diminished in myxedema (6). These improve with replacement therapy. There also may be skeletal muscle weakness contributing to respiratory failure. Testing for maximal inspiratory pressure (MIP) and maximal expiratory pressure (MEP) show reduced strength which improves with treatment of the hypothyroidism. Impaired diffusion capacity, and obstructive sleep apnea are both reversible respiratory manifestations of hypothyroidism (7).

\section{References}

1. Dutta P, Bhansali A, Masoodi SR, Bhadada S, Sharma N, Rajput R. Predictors of outcome in myxoedema coma: a study from a tertiary care centre. Crit Care 2008;12:R1. [CrossRef] [PubMed]

2. Beyon J, Akhtar S, Kearney T. Predictors of outcome in myxoedema coma. Crit Care. 2008;12(1):111. [CrossRef] [PubMed]

3. Klubo-Gwiezdzinska J, Wartofsky L. Thyroid emergencies. Med Clin North Am. 2012;96(2):385-403. [CrossRef] [PubMed]

4. Mathew V, Misgar RA, Ghosh S, Mukhopadhyay P, Pandit K, Mukhopadhyay S, Chowdhury S. Myxedema coma: a new look into an old crisis. J Thyroid Res. 2011;2011:493462.

5. Persani L. Central Hypothyroidism: Pathogenic, diagnostic, and therapeutic challenges. J Clin Endocrinol Metab. 2012:97:3068-3078. [CrossRef] [Pubmed]

6. Zwillich CW, Pierson DJ, Hofeldt FD, Lufkin EG, Weil JV. Ventilatory control in myxedema and hypothyroidism. N Engl J Med. 1975;292(13):662-5. [CrossRef] [PubMed]

7. Schlenker EH. Effects of hypothyroidism on the respiratory system and control of breathing: Human studies and animal models. Respir Physiol Neurobiol. 2012;181(2):123-31. [CrossRef] [PubMed] 